\title{
Modulations in the offspring gut microbiome are refractory to postnatal synbiotic supplementation among juvenile primates
}

\author{
Ryan M. Pace ${ }^{1 \dagger}$, Amanda L. Prince ${ }^{1 \dagger}$, Jun Ma ${ }^{1,3+}$, Benjamin D. W. Belfort ${ }^{1}$, Alexia S. Harvey ${ }^{1}$, Min Hu', \\ Karalee Baquero ${ }^{4}$, Peter Blundell ${ }^{4}$, Diana Takahashi ${ }^{4}$, Tyler Dean ${ }^{4}$, Paul Kievit ${ }^{4}$, Elinor L. Sullivan ${ }^{4,5}$, \\ Jacob E. Friedman ${ }^{6}$, Kevin Grove ${ }^{4}$ and Kjersti M. Aagaard ${ }^{1,2,34^{*}}$
}

\begin{abstract}
Background: We and others have previously shown that alterations in the mammalian gut microbiome are associated with diet, notably early life exposure to a maternal high fat diet (HFD). Here, we aimed to further these studies by examining alterations in the gut microbiome of juvenile Japanese macaques (Macaca fuscata) that were exposed to a maternal HFD, weaned onto a control diet, and later supplemented with a synbiotic comprised of psyllium seed and Enterococcus and Lactobacillus species.

Results: Eighteen month old offspring $(n=7)$ of $36 \%$ HFD fed dams were fed a control ( $14 \%$ fat) diet post weaning, then were synbiotic supplemented for 75 days and longitudinal stool and serum samples were obtained. All stool samples were subjected to 165 rRNA metagenomic sequencing, and microbiome profiles and serum lipids and triglycerides were compared to untreated, healthy age matched and diet matched controls $(n=7)$. Overall, 16S-based metagenomic analysis revealed that supplementation exerted minimal alterations to the gut microbiome including transient increased abundance of Lactobacillus species and decreased abundance of few bacterial genera, including Faecalibacterium and Anaerovibrio. However, serum lipid analysis revealed significant decreases in triglycerides, cholesterol, and LDL $(p<0.05)$. Nevertheless, supplemented juveniles challenged 4 months later were not protected from HFD-induced gut dysbiosis.

Conclusions: Synbiotic supplementation is temporally associated with alterations in the gut microbiome and host lipid profiles of juvenile Japanese macaques that were previously exposed to a maternal HFD. Despite these presumptive temporal benefits, a protective effect against later HFD-challenge gut dysbiosis was not observed.
\end{abstract}

Keywords: Probiotic, synbiotic, Non-human primate, Microbiome, Dysbiosis

\section{Background}

Initial studies of the human microbiome characterized the diversity of the healthy gut microbiome, and subsequent analyses have revealed that the gut microbiome is significantly altered in association with obesity, diet, and metabolic disease [1-9]. Similar trends have been

\footnotetext{
* Correspondence: aagaardt@bcm.edu

${ }^{\dagger}$ Equal contributors

${ }^{1}$ Department of Obstetrics and Gynecology, Division of Maternal-Fetal Medicine, Baylor College of Medicine, Houston, TX 77030, USA

${ }^{2}$ Department of Molecular and Cell Biology, Baylor College of Medicine, Houston, TX 77030, USA

Full list of author information is available at the end of the article
}

observed in animal model systems [10-17], including Japanese macaques (Macaca fuscata) [18]. We have previously demonstrated that the gut microbiome of the Japanese macaque is structured similarly to the human gut microbiome and is modulated primarily by diet, which provides an animal model for examining the human microbiome [18]. However, while diet is associated with significant alterations in the gut microbiome $[4-9,12-17,19]$, duration and modulation of the offspring gut dysbiosis associated with maternal high-fat diet (HFD) exposure is relatively unexplored. Similarly, while no human studies to date have shown causation 
of metabolic disease by the gut microbiome, there have been numerous studies associating dysbiosis of the gut microbiome with metabolic disease [2, 3, 11, 12, 20-23]. We have previously shown that exposure to a maternal HFD during gestation and lactation is associated with impaired islet vascularization, increases in hepatic lipids, and dysbiosis of the offspring gut microbiome at 1 year of age $[18,24,25]$. Altogether, this model provides a unique opportunity to closely examine interactions between dietary exposures, metabolic disease, and the microbiome.

Probiotics are any viable microorganisms (e.g. Lactobacilli and Bifidobacteria) that when consumed or applied confer beneficial effects to the host [26, 27]. Prebiotics are non-host digestible food substances that act as an energy source for beneficial microorganisms in the gut and encourage microbial colonization, growth, and activity [27]. Synbiotics are a combination of the two. These classes of food products are largely accepted as a non-harmful means to promote metabolic health [27]. Previous studies have indicated that prebiotic, probiotic, and synbiotic supplemental use are associated with alterations in physiologic and metabolic measures of host and/ or microbe, bowel function, mucosal contact time, and microbial abundances [28-36]. More specifically, a study with rats demonstrated decreases in weight gain, blood glucose and insulin, and liver triglycerides associated with synbiotic supplementation [33]. Inherent to this notion of benefit is the assumption that the gut is colonized by these probiotic strains and species, although evidence demonstrating either transient or persistent colonization is lacking.

We hypothesized that synbiotic supplementation may modulate the gut microbiome of offspring exposed to a maternal HFD in our non-human primate model (NHP) [18, 37-39]. To test this hypothesis, we utilized a synbiotic composed of probiotic species of Enterococcus and Lactobacillus and a prebiotic of psyllium seed. Psyllium seed (Plantago ovata), is composed mainly of a highly branched arabinoxylan [40], and is a prevalent prebiotic $[35,41,42]$. Psyllium seed is not absorbed into the bloodstream; instead, it passes through the gastrointestinal tract and binds with water to increase stool bulk. Thus, psyllium seed is used to treat diarrhea and diarrhea-like symptoms by absorbing and retaining water in order to make stool more formed and less fluid [43, 44]. In concordance, psyllium seed has been used clinically with diarrhea, irritable bowel syndrome, and inflammatory bowel diseases (e.g. ulcerative colitis and Crohn's disease; reviewed in $[45,46])$. Here, we aimed to determine in our NHP model if synbiotic use leads to transient alterations in the gut microbiome and serum lipid profiles [37-39]. Additionally, and in accordance with our NHP maternal model of obesity, we aimed to examine if synbiotic treatment modulated dysbiosis of the gut associated with a short term HFD challenge at 2 years of age and following 18 months of control diet feeding.

\section{Methods \\ Experimental design}

The use of Macaca fuscata by our group of investigators has been previously described [18, 37-39, 47-50]. In brief, animals were socially housed within indoor and outdoor enclosures at the Oregon National Primate Research Center (ONPRC). All methods were carried out in accordance with IACUC guidelines and regulations, and all experimental protocols were approved by the IACUC at both ONPRC and Baylor College of Medicine. Dams were maintained on a high-fat diet (HFD) consisting of $36 \%$ fat (from porcine and poultry fat, corn and fish oil; TAD Primate Diet 5LOP, Test Diet, St. Louis, MO) that is supplemented with calorically dense treats (consisting of Glaxo powder/TAD pellets, peanut butter, honey, banana, and cornstarch). Animals bred naturally, and in the current study cohort (Fig. 1) offspring were maintained on the same HFD as the dams (via lactation) until weaning at 7 months of age. Post weaning, the offspring were switched to an isocaloric control diet consisting of $14 \%$ fat (from soybean oil, Fiber-Balanced Monkey Diet 5052, Lab Diet, St. Louis, MO).

In this opportunistic study, one socially housed troop of juvenile offspring (17-20 months of age) was observed to have intermittent non-infectious loose stools (culture negative, absence of clinical disease including absence of hair and weight loss, social irritability, or appetite loss) but did not experience any weight loss during the duration of the study (Additional file 1: Figure S1). Within 7 days the animals were supplemented with synbiotic therapy. The synbiotic formulation was administered per os over the course of 75 days hidden in daily treats (consisting of banana, yogurt, pumpkin puree, oats, cinnamon, water, honey, gelatin, probiotic, and prebiotic; Fig. 1). The probiotic component of the synbiotic consisted of $5.0 \times 10^{6}-1.0 \times 10^{7}$ CFUs of Enterococcus faecium (SF-273), Lactobacillus acidophilus (LC-322), Lactobacillus plantarum (LA-5), and Lactobacillus casei (CH6072) (Probiocin oral pet gel, Probios). The prebiotic component consisted of 1.0-1.5 g of psyllium seed powder (EquiAid Natural Psyllium Fiber, Phoenix, AZ; Fig. 1). Around 27 months of age, and postsupplementation, juveniles were challenged with a HFD for 36 days. Two juvenile cohorts that were untreated, healthy, age and dietary exposure matched were used for comparative analyses, including one sample set corresponding to the pre-supplementation time point $(n=3$, two males and one female, 15 months old) and another sample set during a HFD challenge time point $(n=4$, all females, 25-27 months old). Animals utilized in this study were not treated with antibiotics at any point during the course of the study. 


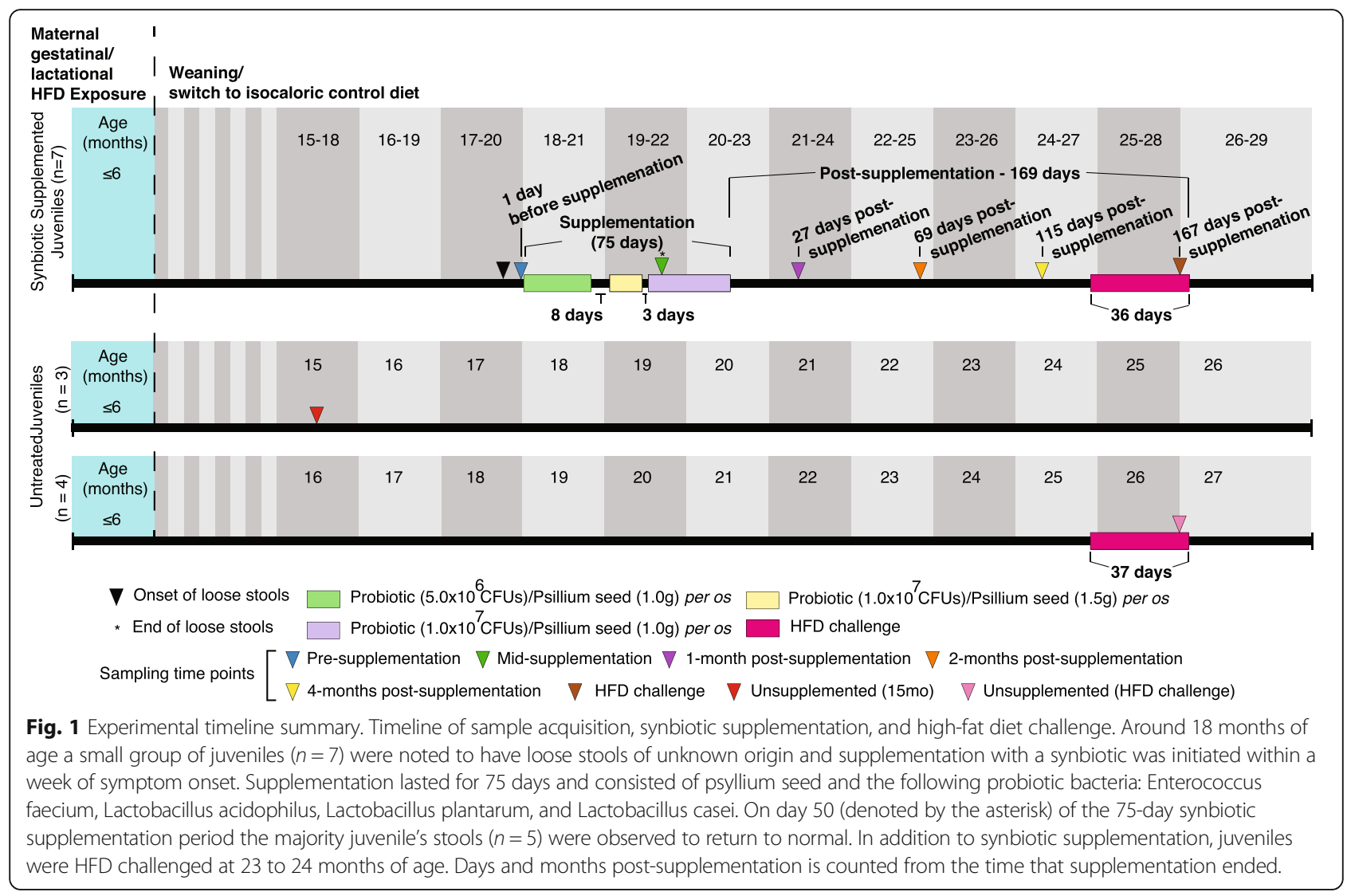

\section{Blood (serum lipid) analysis}

For triglycerides, cholesterol, LDL, and HDL levels, blood samples were taken prior to the start of the synbiotic supplementation and at one-month post-supplementation and submitted for serum lipid profile analysis. Whole-blood samples were collected in red top Vacutainer collection tubes. Serum was separated from whole blood by centrifugation at $2400 \mathrm{rpm}$ for $6 \mathrm{~min}$ (Eppendorf Centrifuge5810, Westbury, New York). Biochemistry and lipid profiles were performed on an ABXPentra400 (Horiba, Irvine, CA) in accordance with manufacturer's guidelines. Quality control procedures were performed each morning prior to analysis with commercial QC materials and samples were run only when the control runs passed inspection. An aliquot of $300 \mu \mathrm{l}$ was analyzed for cholesterol, triglyceride, high- and low-density lipoproteins. Statistical significance was determined using the Wilcoxon matched-pairs signed rank test (non-parametric).

\section{Sequencing of the microbiome}

When animals were individually caged for procedures, samples were collected both as stool and anal collections via cotton swabbing at several time points, including prior to the start of the synbiotic supplementation (17-20 months old, anus), midway through supplementation (stool), and post-supplementation [corresponding to one (stool), two (anus, stool), and 4 months (stool)] and during a HFD challenge that occurred 5 months after treatment (stool, 26-28 months old; Fig. 1, Additional file 2: Table S1). Additionally, samples were collected from the healthy, age and dietary matched, offspring at separate time points: 15 months of age (anal and stool swabs) and during a HFD challenge (anal swabs, 25-27 months old; Fig. 1, Additional file 2: Table S1). Microbial DNA was isolated via the PowerSoil DNA Isolation Kit (MOBIO, Carlsbad, CA). After DNA extraction, microbial 16 SrDNA was amplified using V3 V5 primers with barcodes attached for sample identification before Roche454 sequencing $[18,51]$. The V3 V5 primer sequences used include, with barcodes in lower case letters: 5'-cctatcccetgtgtgccttggcagtctcaGCCTACGGG AGGCAGCAG-3' (B-354F forward primer) and 5'-cca tctcatccctgcgtgtctccgactcagNNNNNCCGTCAATTCM TTTRAGT-3' (A-926R reverse primer).

\section{5 data analysis}

Sample reads were analyzed using quantitative insights into microbial ecology (QIIME, version 1.9.1) [52]. Briefly, reads lacking a barcode or primer sequence, reads with length less than 200 nucleotides, and reads with a minimum average quality score less than 25 were removed. De-noised sequences were clustered into 
operational taxonomic units (OTUs) at the $97 \%$ similarity level through closed-reference OTU picking using the Greengenes 13.5 database [53], resulting in $73 \%$ of sequences $(316,470 / 432,779)$ remaining for subsequent analysis (Additional file 2: Table S1). Microbial diversity was evaluated within samples (alpha diversity) or between samples (beta diversity) on rarefied OTU tables. Rarefaction resulted in 2969 and 3125 sequences from each sample used in the analyses of the intervals containing the synbiotic supplementation and HFD challenge, respectively. Alpha diversity was evaluated using Shannon Diversity Index and chao1. Beta diversity was evaluated using phylogenetic distance (weighted and unweighted UniFrac) [54]. The resulting distance matrices served as inputs for principal coordinates analysis (PCoA) and significance of sample clustering by PERMANOVA with 999 permutations. Phylogenetic investigation of communities by reconstruction of unobserved states (PICRUSt) [55] was used to infer relative abundance of bacterial encoded metabolic pathways, as defined by the Kyoto Encyclopedia of Genes and Genomes (KEGG;http://www.genome.jp/kegg/). Significant differences in bacterial taxa and metabolic pathways between groups was identified via STAMP (White's nonparametric t-test for two groups comparisons) [56] and feature selection through linear discriminant analysis (LDA) effect size (LEfSe) [57]. For LEfSe, the alpha value for the factorial Kruskal-Wallis test was 0.05 and the threshold on the logarithmic linear discriminant analysis (LDA) score for discriminative features was 2.0. Weight and lipid data were analyzed using Prism 6 (GraphPad Software Inc La Jolla, CA). The $16 \mathrm{~S}$ sequence data generated from this analysis have been deposited in the Sequence Read Archive (SRA) under accession bioproject ID PRJNA317339.

\section{Probiotic species-specific PCR}

Primers were selected from previously reported studies $[58,59]$ or designed using Primer-BLAST from the National Center for Biotechnology Information (NCBI) [60]. Primer sequences and annealing conditions are included in Additional file 3: Table S2. Resultant amplicons were run on a $2 \%$ agarose gel and visualized using a GelDoc-it [2] Imager (UVP, LLC Upland, CA). For quantitative real-time PCR (qPCR), the FastSYBRGreen kit (Applied Biosystems ABI, Waltham, MA) was utilized, and the reaction was run on a StepOnePlus PCR System (ABI). The following strains of the probiotic species were used as positive controls for PCR - L. casei SD5868, L. acidophilus ATCC521, and L. plantarum ATCC14917.

\section{Results}

Alterations of the juvenile gut microbiome are associated with synbiotic supplementation

Examining taxonomic differences between untreated juveniles and synbiotic supplemented juvenile offspring (Fig. 1),

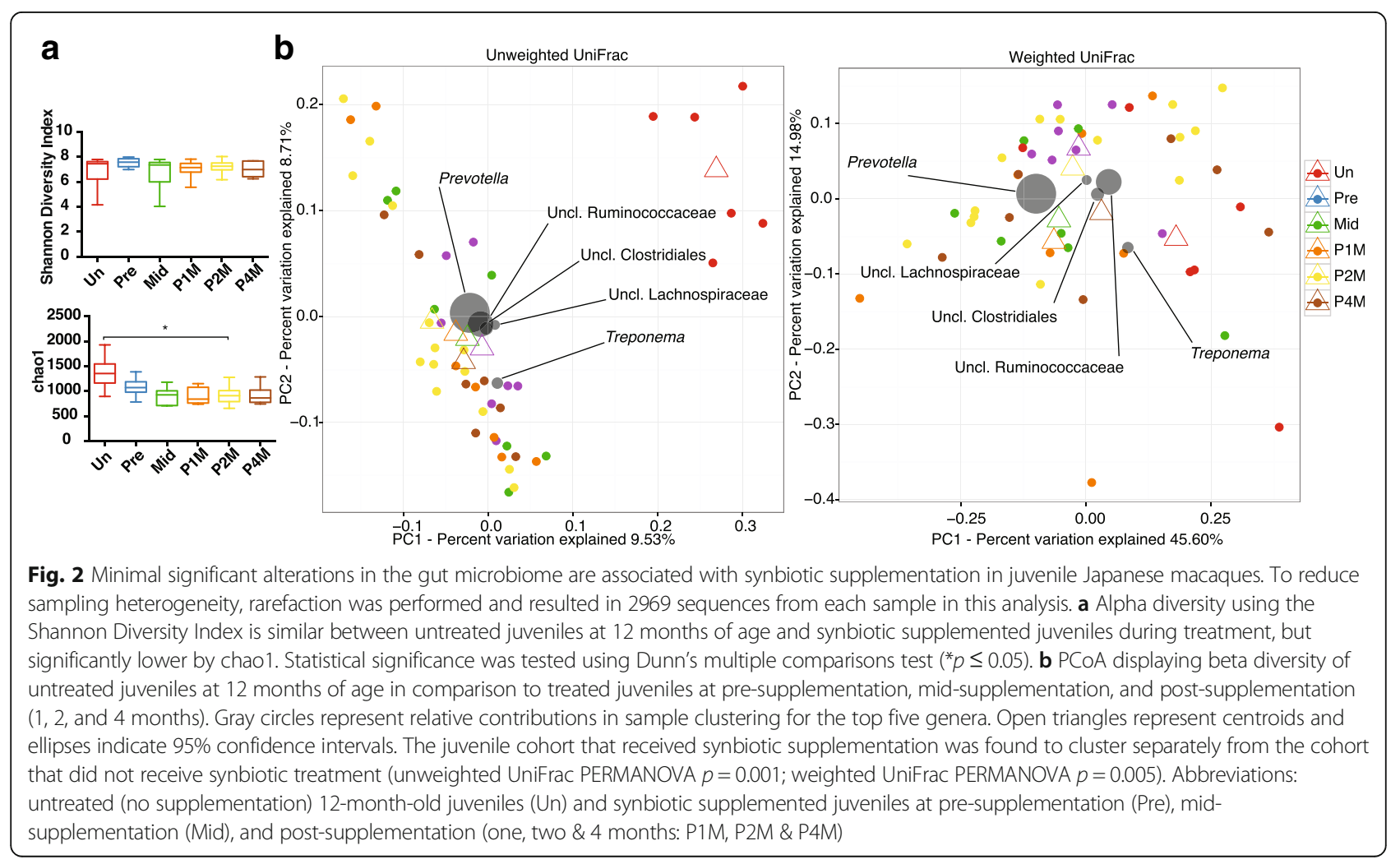


we identified shifts in bacterial taxa (Fig. 2). Examination of alpha diversity measures (i.e., within sample diversity) among offspring that received synbiotic supplementation at pre, mid, and post-supplementation (1, 2 \& 4 months after supplementation ended) indicated unchanged Shannon but significantly lower chao1 metrics (Fig. 2a). By beta diversity metrics, untreated juveniles clustered separately from the synbiotic supplemented juveniles at all stages of synbiotic treatment but not pretreatment (unweighted UniFrac PERMANOVA $p=0.001$; weighted UniFrac PERMANOVA $p=0.005$; Fig. $2 b$, Additional file 4 : Table S3). These could largely be attributed to a few taxa, including Prevotella $(0.17 \%$ in untreated animals and $0.32 \%$ in animals prior supplementation, $p=0.013$ ) and Clostridium (untreated $0.003 \%$ vs pre-supplementation $0.009 \%$, $p=9.28 \mathrm{e}^{-3}$; Fig. 3a, Additional file 5: Figures S2 \& S3). This data is in general agreement with previous reports on the association of Prevotella with high fiber diets $[4,6,19]$, which the psyllium would provide. This is further consistent with our observed significant shifts in the relative abundance at the genus level as identified via LEfSe analyses, including decreases in Faecalibacterium (from 2.46 to $0.63 \%, p=1.97 \mathrm{e}^{-3}$ ), Anaerovibrio (from 0 . $27 \%$ to $\left.0.09 \%, p=8.10 \mathrm{e}^{-4}\right)$, and Phascolarctobacterium (from $0.65 \%$ to $0.15 \%, p=0.021$; Fig. 3b, Additional file 5: Figures S2 \& S3). A decrease in Anaerovibrio is in accordance with a prior study where A. lipolytic was decreased in association with a high-fiber diet [61]. Of note, we did not detect significant changes in the bacterial taxa related to the probiotic species during treatment, including manual inspection of the Enterococcus and Lactobacillus OTU reference sequences (with $>10$ read counts) from the untreated, pre-, mid-, and post-supplementation (Additional file 6: Figure S3B and Additional file 7: Table S4). While manual inspection of these samples revealed three putative probiotic OTUs with blast hits against the
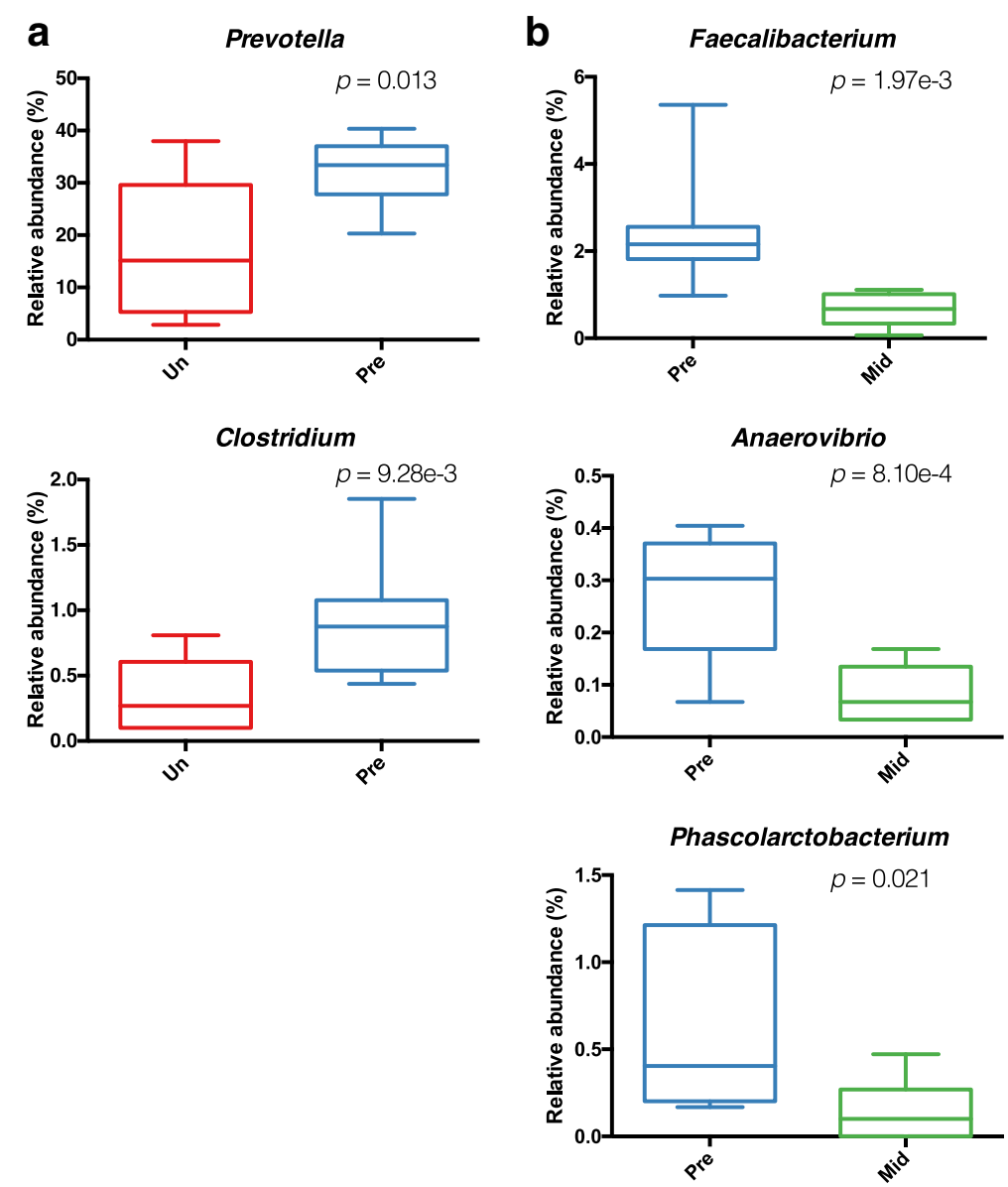

Fig. 3 Synbiotic supplementation is associated with trends in genus level taxonomic changes. LEfSe analysis was utilized at the genus level to identify alterations in taxonomical enrichment between (a) animals that were untreated and prior to synbiotic supplementation, and between (b) animals prior to synbiotic supplementation and during mid-supplementation. Abbreviations as in Fig. 1. Statistical significance was tested using Dunn's multiple comparisons test ${ }^{* *} p \leq 0.01$ ) 
probiotic species, these OTUs were either not detected in the mid-supplementation samples or were not significantly increased compared to pre-supplementation (Additional file 7: Table S4). Furthermore, we were only able to detect one of the four probiotic species (L. acidophilus) in the synbiotic supplemented animals by PCR (Additional file 8: Figure S4) but did detect overall changes in the Lactobacillus genus via quantitative real-time PCR (qPCR, Additional file 9: Figure S5).

\section{Synbiotic supplementation is temporally associated with improvements in serum lipids and associated bacterial metabolic pathways}

Despite the relatively minimal change in bacterial taxa associated with synbiotic supplement, we did find significant alterations in host lipid profiles (Fig. 4). Analysis of serum taken at pre-supplement and one-month postsupplement revealed significant decreases in triglycerides (67.0 to $40.1 \mathrm{mg} / \mathrm{dl}, p=0.02$ ), cholesterol (155.3 to 144 . $1 \mathrm{mg} / \mathrm{dl}, p=0.03$ ), and LDL levels ( 81.7 to $71.3 \mathrm{mg} / \mathrm{dl}, p=$ $0.03)$. In contrast, although lowered, we did not find a significant difference in the level of HDL (70.4 to $68.0 \mathrm{mg} / \mathrm{dl}$, $p=0.28)$. However, despite the statistical significance of these variations, the physiologic and clinical significance is non-evident as the mean variation (in $\mathrm{mg} / \mathrm{dL}$ ) was within $\sim 10 \%$ of initial values. In contrast, serum triglyceride levels demonstrated a higher degree of variance pre and post synbiotic supplementation (59.8\% of the initial value, Fig. 4). Over the course of this study we did not detect any significant changes in the weight of the animals that received synbiotic supplementation at any time

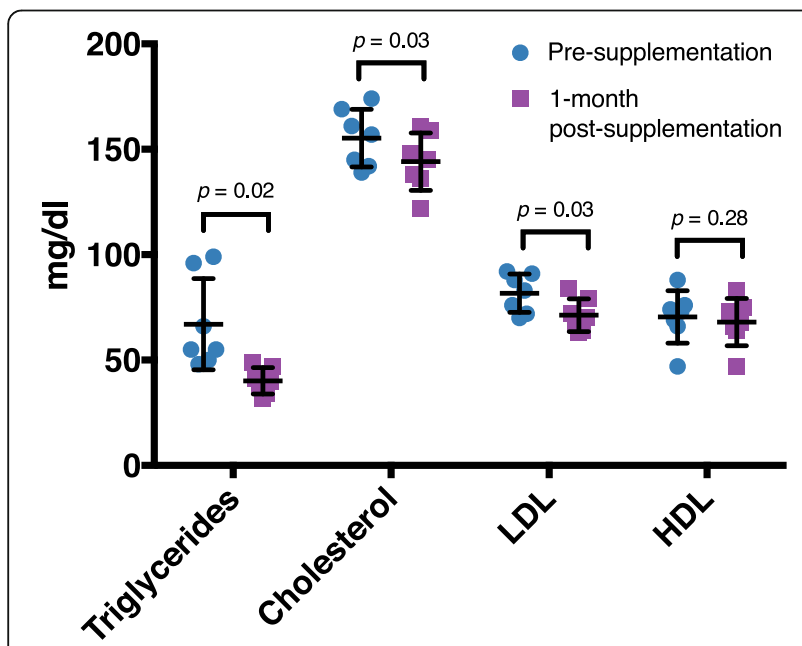

Fig. 4 Host lipid profiles are altered in association with synbiotic supplementation. Juvenile offspring triglycerides, cholesterol, and LDL (but not HDL) profiles were significantly decreased at one-month post-supplementation in comparison to pre-supplementation point prior or during supplementation, nor in comparison to untreated, age and dietary-matched animals (Additional file 1: Figure S1). On day 50 of the 75day synbiotic supplementation the majority of the juveniles ( 5 out of $7 ; 71.4 \%$ ) were observed to have normal, firm stool.

With these temporal lipid modifications in mind, we further examined inferred bacterial metabolic pathways for alterations in association with synbiotic supplementation and did observe significant differences (Fig. 4). Specifically, we observed significant decreases in several lipid metabolism pathways, including fatty acid metabolism $(0.22$ to $0.20 \%, p=0.038)$, alpha-linolenic acid metabolism (0.002 to $\left.0.001 \%, p=5.18 \mathrm{e}^{-3}\right)$, and synthesis and degradation of ketone bodies $(0.04$ to $0.03 \%, p=1$. $30 \mathrm{e}^{-3}$; Fig. 5, Additional file 10: Figure S6). In contrast, the only metabolic pathways that we observed as enriched during synbiotic supplementation were for sphingolipid metabolism ( 0.19 to $0.22 \%, p=0.080)$ and glycosphingolipid biosynthesis-globo series (0.12 to 0 . $14 \%, p=0.070$; Fig. 5, Additional file 10: Figure S6).

\section{Synbiotic treatment does not confer protection from HFD-associated gut dysbiosis following later challenge} Nineteen weeks after the synbiotic supplementation ended, juveniles were challenged for 1 month with the $36 \%$ HFD that they had been previously exposed to via their mothers (during both gestation and lactation; Fig. 1). Overall, we observed significant differences in the chao1 but not Shannon alpha diversity measure between groups (Fig. 6a), alongside both unweighted and weighted UniFrac beta diversity (unweighted UniFrac, PERMANOVA, $p=0.001$; weighted UniFrac, PERMANOVA, $p=0.024$; Fig. 6b, Additional file 11: Table S5). Deeper taxonomic interrogations via LEfSe revealed several non-significant differences in the relative abundance of bacterial taxa before and during the HFD challenge. Bacteroidales (Bacteroidetes), Fibrobacteres, and Spirochaetes were not significantly reduced ( 38.9 to 32.3 [ $p=0.677], 0.53$ to 0 $\left[p=4.67 \mathrm{e}^{-3}\right], \quad 8.2$ to $2.4 \%[p=0.078]$, respectively), whereas Tenericutes and Erysipelotrichi were significantly increased ( 0.3 to $2.5 \%$ [ $p=0.014], 0.28$ to $16.2 \%$ [ $p=8$. $\left.82 \mathrm{e}^{-4}\right]$, Additional file 12: Figure S7). Additionally, and consistent with our prior findings [18], classes of Proteobacteria were non-significantly differentially abundant in association with the postnatal HFD challenge: Epsilonproteobacteria were non-significantly enriched from 0.009 to $0.99 \%$ ( $p=0.150$ ), whereas Deltaproteobacteria were non-significantly reduced from 0.26 to $0.01 \%$ $(p=0.086)$ (Additional file 12: Figure S7). Although synbiotic supplementation did not inhibit HFD-associated gut dysbiosis, there were a number of significant differences in comparison to animals that received a HFD challenge but no synbiotic supplementation prior (Fig. 7a, b). 


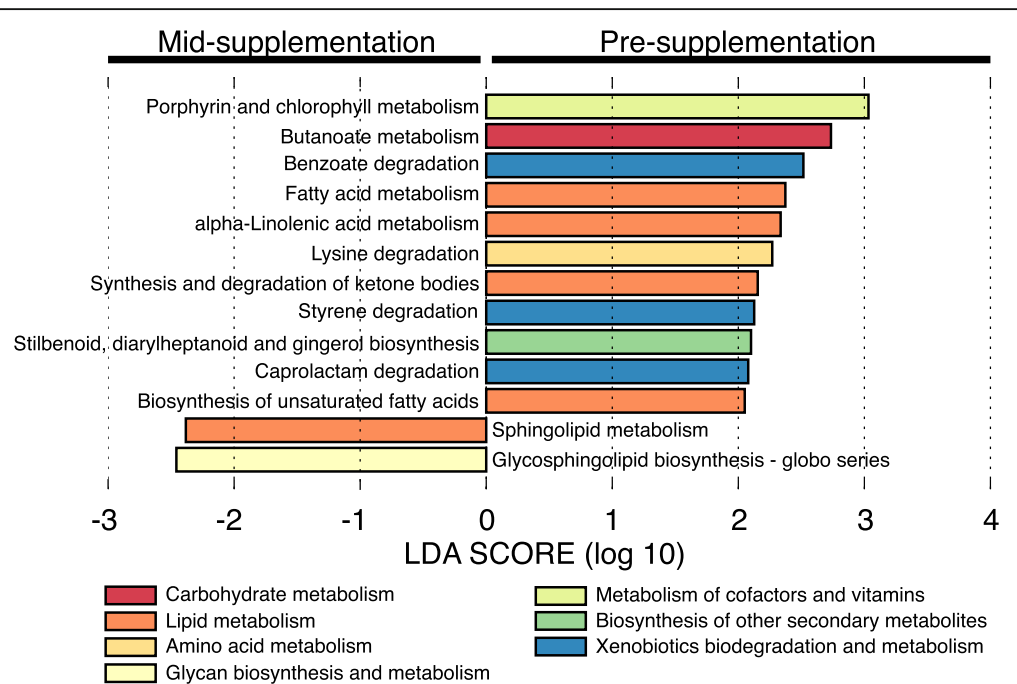

Fig. 5 Shifts in inferred metabolic pathways are associated with synbiotic supplementation. LEfSe plot of inferred KEGG bacterial metabolic pathways with differential abundance. Bars to the left indicate inferred bacterial metabolic pathways that are enriched in animals during synbiotic supplementation, while bars to the right indicate inferred bacterial metabolic pathways that are enriched in animals during pre-supplementation. Bar colors indicate higher-level metabolic pathways

In particular, there was a non-significant increased abundance of taxa from Bacilli (Streptococcus 2.61 to $0.06 \%, p$ $=0.102]$ ), Erysipelotrichi $(0.11$ to $0 \% p=0.06])$; significance was uniquely observed in Bulleidia (2.46 to $0.63 \% p$ $\left.=6.49 \mathrm{e}^{-3}\right)$. The non-significant increase in
Erysipelotrichi and decrease in Fibrobacteres appear only among pre/prior synbiotic supplementation state (Additional file 12: Figure S7C). This is particularly potentially intriguing with regard to Eubacterium as this genus has been previously associated with obesity and a

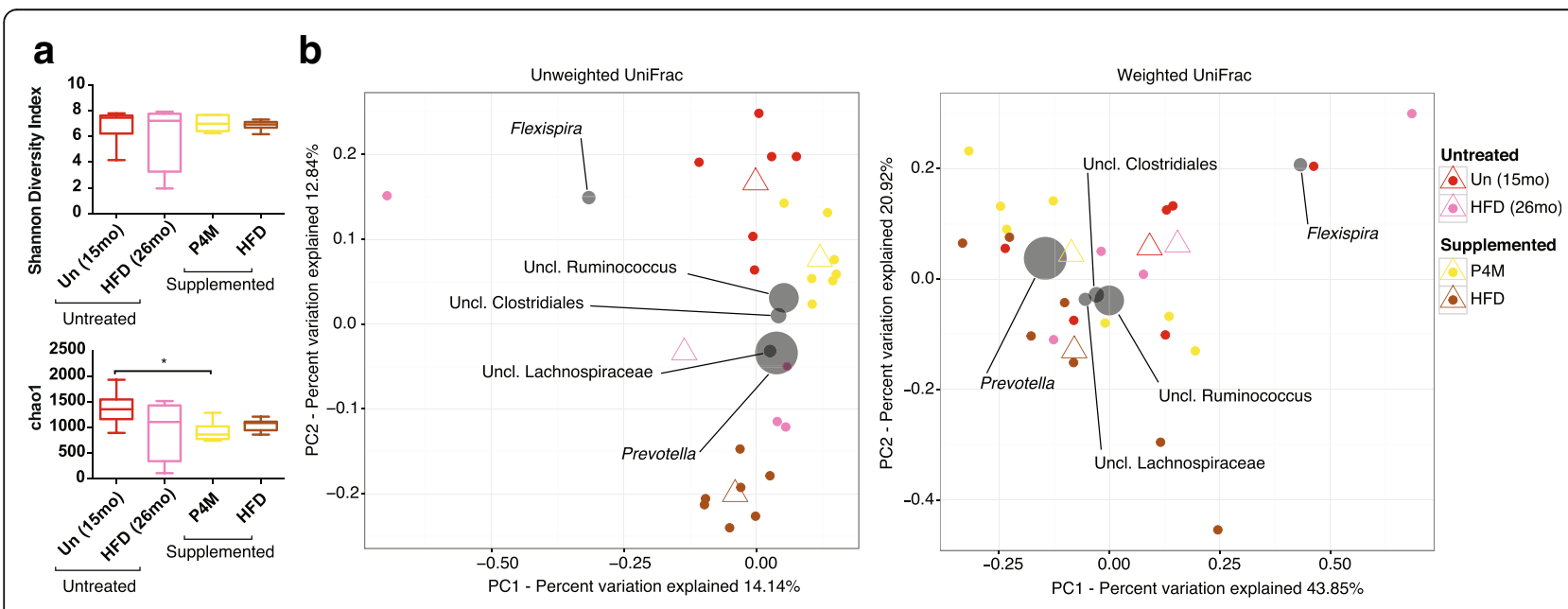

Fig. $6 \mathrm{HFD}$ challenge is associated with alterations in the gut microbiome. To reduce sampling heterogeneity, rarefaction was performed utilizing 3125 sequences from each sample. a Alpha diversity of synbiotic supplemented juveniles at 4-months post-supplementation and HFD challenge, and untreated juveniles at 15 months and during HFD challenge (26 months). 4-months post-supplementation juveniles were found to have a significant lower alpha diversity compared to untreated juveniles as measured by chao1 but not by Shannon. Statistical significance was tested using Dunn's multiple comparisons test. Asterisk indicates a $p \leq 0.05$. b PCoA displaying beta diversity (unweighted and weighted UniFrac) of synbiotic supplemented juveniles at 4-months post-supplementation, HFD challenge, and untreated juveniles at 15 months and during HFD challenge (26 months). Gray circles represent relative contributions in sample clustering for the top five genera. Open triangles represent centroids and ellipses indicate $95 \%$ confidence intervals. All groups were found to significantly cluster different from one another (unweighted UniFrac PERMANOVA, $p=0.001$; weighted UniFrac PERMANOVA, $p=0.024$ ). The juvenile cohort that received synbiotic supplementation was found to cluster separately from the cohort that did not receive synbiotic supplementation (unweighted UniFrac PERMANOVA, $p=0.001$; weighted UniFrac PERMANOVA, $p=0.022$ ). Abbreviations: Un (15mo): untreated juveniles at 15 months; HFD (26mo): untreated juveniles during HFD challenge at 26 months; P4M: treated juveniles at 4-months post-treatment; HFD: treated juveniles during HFD challenge 


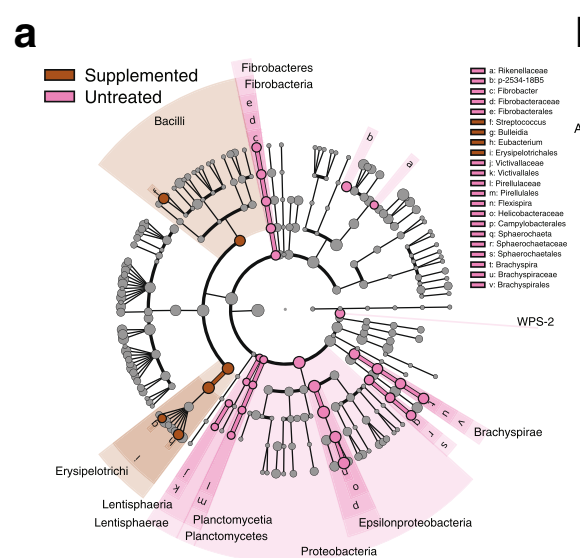

b
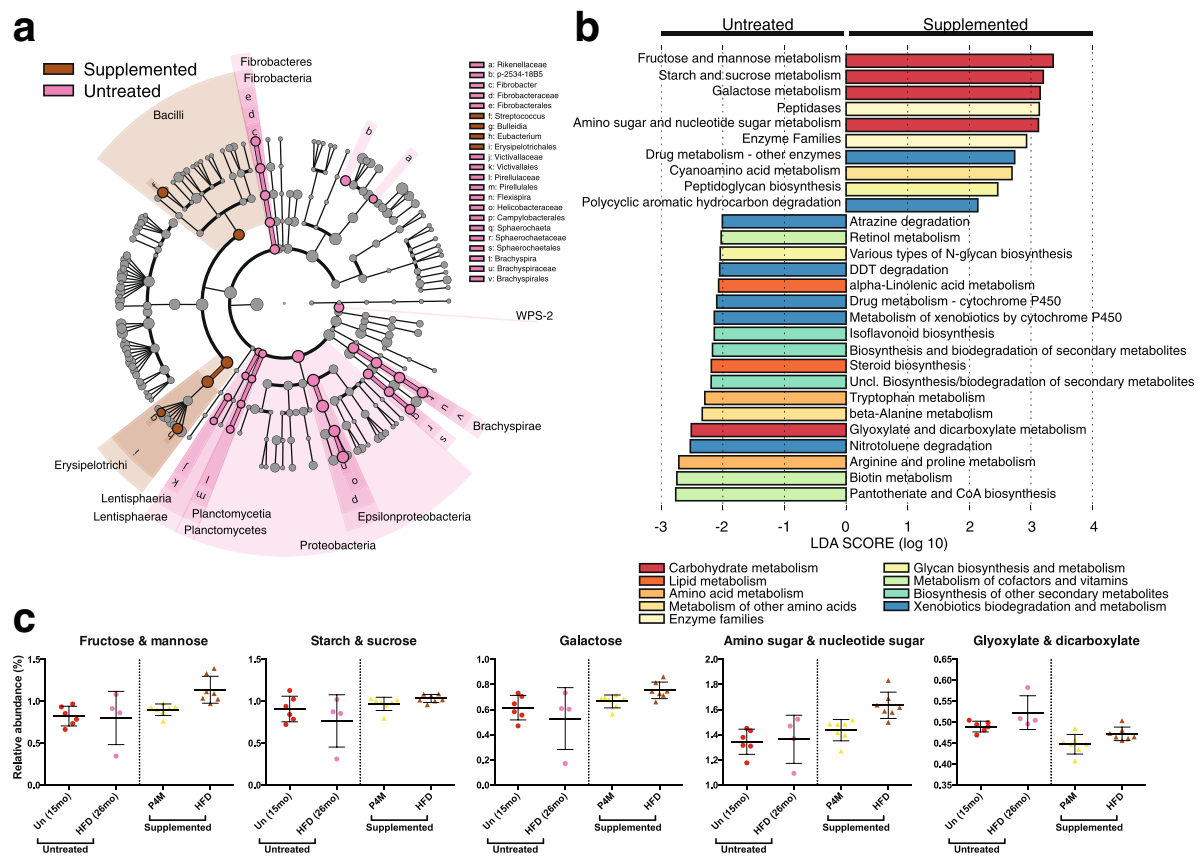

Fig. 7 Prior synbiotic supplementation is associated with differences in bacterial taxa and inferred metabolic pathways during HFD challenge. a LEfSe generated cladogram of taxonomic differences of abundance. Areas shaded purple indicate bacterial taxa with a higher abundance in prior supplemented animals during HFD challenge, whereas areas shaded green indicate bacterial taxa with a higher abundance in untreated animals during HFD challenge. $\mathbf{b}$ LEfSe plot of inferred KEGG bacterial metabolic pathways. Bars to the left indicate inferred bacterial metabolic pathways enriched in untreated animals during HFD challenge, whereas bars to the right indicate inferred bacterial metabolic pathways enriched in prior supplemented animals during HFD challenge. Bar colors indicate higher-level metabolic pathways. c Plots of the relative abundance of differentially enriched carbohydrate metabolism pathways identified via LEfSe analysis of prior supplemented versus untreated animals during HFD challenge. Abbreviations used as in Fig. 4

HFD [62]. Furthermore, during the HFD challenge we found that prior synbiotic supplemented juvenile had an enrichment of pathways for carbohydrate metabolism and enzyme families and reduced enrichment of pathways for xenobiotics biodegradation and metabolism and metabolism of cofactors and vitamins in comparison to untreated juveniles (Fig. 7b, c, Additional file 13: Figure S8).

\section{Discussion}

Overall, we found that there were observable and a few significant alterations in the gut microbial community structure and function (e.g., gut ecology) in temporal association with synbiotic use. These microbiome rearrangements associated with synbiotic supplementation included several shifts in taxonomic relative abundances during synbiotic supplementation which have been associated with host biology. For example, we saw a decrease in Faecalibacterium, and F. prausnitzii has been associated with anti-inflammatory properties in Crohn's disease and in animal models of colitis [63, 64]. Similarly, we observed a decrease in the relative abundance of Phascolarctobacterium, which has been shown increase in relative abundance in rats that received a HFD compared those on control diet [14]. Lastly, we saw a decrease in Anaerovibrio, which is in agreement with a previous study in Surti buffalo associating gut ecology and dietary fiber $[61,65]$.

Based on the limited but observable and significant taxonomic shifts observed during synbiotic supplementation, it is not surprising that we observed statistically significant concurrent decreases in cholesterol, triglyceride, and LDL levels (Fig. 4). The results of our lipid profiles prompted us to examine the inferred metabolic pathways of the gut microbiome in greater detail, and provide microbiome community function data which correlates well with our serum lipid measurements. Consistent with both our microbiota and host lipid data, we found a decrease in fatty acid metabolism pathway gene content in association with synbiotic exposure (Fig. 5). These data are in concordance with prior studies in rats and humans that have found synbiotic consumption leads to decreased levels of serum cholesterol and triglycerides [66, 67]. However, while we were unable to determine if there was a higher abundance of catabolic versus anabolic pathways, our prior work in this model has shown that lipid metabolism pathways are positively correlated to bacteria, including Campylobacter and Runimococcus [18]. In the current study, we observed enrichment for sphingolipid metabolism and glycosphingolipid biosynthesis-globo series 
pathways during synbiotic supplementation. Interestingly, this metabolic pathway data is consistent with previous work of others demonstrating that treatment with a Bacteroides derived glycosphingolipid in gnotobiotic mice regulates colonic invariant natural killer $\mathrm{T}$ cell (iNKT) homeostasis to protect against oxazolone induced colitis [68]. Taken together, our findings and those of others suggest that synbiotic supplementation may act as an ecological modulator of the gut microbial community, rather than via direct xenobiosis.

Despite these presumptive beneficial findings during and immediately post synbiotic supplementation, we failed to observe persistent significant metabolic protection from gut dysbiosis following later HFD challenge. However, we did observe several changes in bacterial taxa at the genus level between synbiotic treated animals to untreated animals during HFD challenge which are worth commenting on. The lower relative abundance of Fibrobacter is likely related to the HFD, as F. succinogenes is a well-known degrader of cellulose [69], and cellulose is a principal component of the Japanese macaque diet. The remaining genera that were observed to have a lower relative abundance in synbiotic supplemented animals have been previously associated with various forms of gastrointestinal distress. Brachyspira are associated with intestinal spirochetosis and diarrhea [70], and Sphaerochaeta, related to Brachyspira, have been shown to have highly chimeric genomes, sharing more than 40 $\%$ of the total number of its genes with Clostridia [71]. Flexispira, reclassified as members of Helicobacter [72], are associated with colitis in humans [73]. The bacteria with increased relative abundance in the gut microbiome of synbiotic-supplemented juveniles include Bulleidia, Eubacterium, and Streptococcus. Bulleidia extructa is an obligate anaerobe that has been described in association with periodontal disease [74]. While B. extructa is described as an oral pathogen, it is related to Holdemania filiformis and Erysipelothrix rhusiopathiae, which are both found in the stool [74]. Therefore, it's possible that a species of Bulleidia also resides in the gut, which is supported by a study examining the microbiome of the appendix [75]. In this study, the relative abundance of Bulleidia was increased in subjects with appendicitis [75]. While Bulleidia has been associated with disease, it was found in the appendix and rectum of healthy subjects although at a low relative abundance [75]. Eubacterium is also associated with host disease and has been found to be increased in coronary heart disease subjects with metabolic syndrome [62]. While Eubacterium are one of the most abundant group of human colonic butyrate producers [76], we did not observe a significant difference in the predicted pathway for butyrate metabolism during the HFD challenge. Streptococcus species have been implicated in both detrimental and beneficial aspects of non-alcoholic fatty liver disease (NAFLD) [67, 77], and we have previously extensively described that both juveniles and fetuses exposed to a maternal HFD have NAFLD in our Japanese macaque model [25, 38, 47, 78]. While a strain of $S$. mutans has been shown to aggravate NAFLD in mice [77], S. thermophilus in a probiotic cocktail mixture that included Lactobacillus and Bifidobacterium species was shown to ameliorate NAFLD induced by a HFD in rats [67]. While our synbiotic cocktail did not include Streptococcus species, we speculate that our findings are consistent with the host having multiple body site-specific niches where one microbe supplementation (or loss) subsequently disrupts the relative abundance of another $[1,51,79-81]$.

Accompanying these alterations in several taxa occurring in association with the HFD challenge were modulations in their inferred bacterial metabolic pathways. For example, expression of a glyoxylate shunt in mice resulted in resistance to the associated effects of HFD consumption, such as improved fat mass, leptin, and plasma triglycerides [82]. While the pathway for glyoxylate metabolism was not enriched in the supplemented animals compared to non-supplemented animals, it was increased in response to the HFD challenge in both groups (Fig. 7c). In further agreement, a prior study with rats provided a high prebiotic fiber diet (derived from inulin and oligofructose) post-weaning were shown to have a dampened response to the negative effects associated with HFD consumption (e.g., lower ratio of Firmicutes to Bacteroidetes and decreased cholesterol) [19]. These results were considered to be due to the fiber affecting alterations in the gut microbiota and hepatic gene expression [19]. Taken together, our observations lead us to speculate that the altered carbohydrate metabolic pathways are most likely attributable to the establishment of a microbial community during the synbiotic supplementation that more readily produces short chain fatty acids (SCFAs) [83]. Although we did not measure SCFA production directly, it is well-documented that psyllium alters SCFA production [84] and thereby lipid metabolism [85], leading to an altered response of the microbiome in association with the HFD challenge in the animals that received prior synbiotic supplementation. While we are able to speculate on potential synbiotic and HFD gut taxonomic and functional pathway associations as identified by metagenomic sequencing, future in-depth studies are needed.

There are several limitations to our study. First, it was a convenience study precipitated by the use of probiotics for loose and non-infectious stools. As such, it is limited by small sample size but benefits from pairwise study design. We observed significant differences in clinically relevant measures during synbiotic supplementation, suggesting our small sample size was 
nevertheless adequately powered. Similarly, following high fat diet challenge, we observed significant differences in chao 1 but not Shannon diversity measures. Given observed significance in some but not all measures, our findings most likely reflect the underlying biology and support the notion that synbiotic supplementation is not associated with persistent changes in the gut ecology. Second, we could not detect the probiotic strains in post-supplementation stool samples. However, this inability to detect the bacterial strains in the synbiotic per se are both consistent with and in contrast to previous human studies [29, 31, 34, 35, 41], and may be due to several reasons, such as synbiotic dose, probiotic species, and model system. For example, prior studies in humans have utilized Bifidobacterium [29, 31, 34, 35, 41], which differs from the probiotics used in this study. Thus, the inability to detect strains or species may be due to the absence of Bifidobacteria in the synbiotic supplement. However, we were able to detect alterations in Lactobacillus at the genus level in supplemented juveniles (Additional file 1: Figures S4 and S5). This lack of detection at the species level is consistent with other studies, and may be due to the complexity of the sample, the probiotic dosage, and/or model system. A prior study in canines where bacterial DNA from a probiotic was detected by quantitative real-time PCR (qPCR) provided daily supplementation at $>10^{8} \mathrm{CFU}[86]$. In contrast and consistent with our findings, a human study employing culturable strains similarly could only detect later stool output by qPCR at the genus level [87]. More recently, a study in preterm infants was able to detect probiotic strain DNA by qPCR but only in neonatal stool [88], which is non-complex and harbors similar strain microbes found within the NICU [89]. Therefore, the ability to detect probiotic bacterial DNA in the stool of preterm infants may be due a lower complexity of the sample or shared built environments.

Despite these limitations, our study has several strengths. First, it is a "real world" probiotic evaluation, whereby initial use followed common indications in both veterinary and human populations (namely, loose stools). Second, despite our inability to detect our probiotics strains (but demonstrated ability to detect same-strain genus increased abundance), we have observed significant improvement in lipid profiles concomitant with microbiome rearrangement and prokaryotic pathway enrichment unique to our synbiotic supplemented animals and only during supplementation. Thus, our data suggest that the probiotic mechanisms are likely through temporal ecological modulation rather than direct effects. Third, we leveraged an exceedingly well characterized primate model and were thus able to control for many potential confounders.

\section{Conclusions}

Collectively, our findings in our primate model of maternal HFD feeding indicate that synbiotic supplementation is temporally associated with minimal and a few significant alterations in the gut microbiome of juvenile offspring. Overall, the majority of microbiome alterations in both beta and alpha diversity were not significant. However, synbiotic supplementation was affiliated with significant changes in the enrichment of specific carbohydrate pathways that may be tied to microbial production of SCFAs and host lipid profiles associated with both a maternal HFD and later in life HFD challenge [90]. Nevertheless, a protective effect against further gut dysbiosis associated with a HFD challenge at 2 years of age was not observed. Altogether, our data suggest that while synbiotic supplementation is associated with a few significant modulations of the microbiome and functional benefits to host physiology, this synbiotic-altered gut ecology does not persist and is refractory to later HFD feeding. These findings further support our prior observations that HFD exposure, be it solely maternal or as a later in life challenge, remains a significant driver of alterations of the gut microbiome. This lasting footprint of the maternal diet persists, and cannot be beneficially modified in the two years postnatally by neither synbiotic supplementation nor control diet feeding from the time of weaning onward.

\section{Additional files}

Additional file 1: Figure S1. Loose stools and synbiotic

supplementation was not associated with significant weight loss. Juveniles were supplemented with synbiotics after experiencing loose stools $(n=7)$. To determine if weight loss following symptoms and synbiotic supplementation occurred, we compared the weight of the synbiotic supplementation cohort with an untreated, healthy, agematched cohort $(n=4)$ that was also matched for dietary exposures. Measurement of body weight was performed using the Ohaus ES series scale (Parsippany, NJ). There was no significant difference in the mean weight of synbiotic supplemented juveniles in comparison to untreated juveniles from prior, during, and post-supplementation. Error bars represent standard deviation. Test for significance was performed using Sidak's multiple-comparisons test. (PDF $132 \mathrm{~kb}$ )

Additional file 2: Table S1. Sample/count summary. Provided are sequencing read counts (minimum, median, max, and mean) alongside sex, age and number of samples for each animal. SD = standard deviation. (PDF $24 \mathrm{~kb}$ )

Additional file 3: Table S2. Primers used in probiotic species-specific PCR. Table of primers used for PCR identification of probiotic species and/or genus. (PDF $21 \mathrm{~kb}$ )

Additional file 4: Table S3. Unweighted and weighted UniFrac PERMANOVA $p$-values of samples from untreated (15mo), pre-, mid-, and post-supplementation juveniles (1, 2, and 4 months after the end of supplementation). (PDF $12 \mathrm{~kb}$ )

Additional file 5: Figure S2. Bacterial taxa altered in association with synbiotic supplementation. LEfSe plot of bacterial taxa with differential abundance. Areas to the right and shaded orange indicate bacterial taxa with a higher abundance in animals from the pre-supplementation group, whereas areas to the left and shaded red indicate bacterial taxa 
with a higher abundance in animals from the untreated (15 months) group. (PDF $342 \mathrm{~kb}$ )

Additional file 6: Figure S3. Taxonomic shifts associated with synbiotic supplementation. A. LEfSe plot of bacterial genera with an increased relative abundance during pre-supplementation. LEfSe did not detect an enrichment of bacterial taxa during mid-supplementation. B. Examination of the relative abundance of probiotic strains from pre-supplementation to 4-months post-supplementation revealed no significant changes. Statistical significance was tested using Dunn's multiple comparisons test. (PDF $406 \mathrm{~kb}$ )

Additional file 7: Table S4. BLAST results for OTU representative sequences. (XLSX $12 \mathrm{~kb})$

Additional file 8: Figure S4. Majority of species-level bacterial DNA from probiotics is undetectable in the gut of supplemented juveniles. DNA was isolated from anal swabs and stool

samples collected at pre-, mid-, post-supplementation. PCR was performed to detect probiotic bacterial DNA at the species level (Enterococcus faecium, Lactobacillus plantarum, L. casei, and L. acidophilus). L. acidophilus was the only probiotic bacterial species that was detected at any time point assayed, and only detected in 2 animals (animal 31,267 and faint band in 31,093). A 165 universal PCR was performed to ensure the presence of bacteria within the samples, and species specific positive controls were run alongside blank negative controls (far right panel). $n=$ 7 subjects per time point. (PDF $19 \mathrm{~kb}$ )

Additional file 9: Figure S5. Lactobacillus genera are quantitatively increased in the gut of synbiotic-supplemented juveniles. DNA was isolated from anal swabs and stool samples collected at pre-, mid-, postsupplementation. PCR was performed to detect Lactobacillus at the genus level. (A) While bacterial DNA from probiotics was undetectable at the species level in the gut of supplemented juveniles, we were able to detect Lactobacillus in the gut at the genus level in both pre-, mid, and post supplemented juveniles. (B) Increases in Lactobacillus at the genus level was detectable by quantitative real-time PCR (qPCR). (C) The majority of supplemented juveniles (5/7) had a detectable increase in Lactobacillus at the genus level by $\mathrm{QPCR}$, which resolved post-supplementation. $n=7$ subjects per time point. (PDF $5089 \mathrm{~kb}$ )

Additional file 10: Figure S6. Relative abundance of LEfSe-identified enriched KEGG pathways in the pre-supplemented and mid synbiotic supplemented groups. STAMP generated two groups extended error bar plot. Plots on the left indicate relative abundance of enriched KEGG pathways. Plot on the right indicates the difference in the mean proportion. Statistical significance was tested using White's non-parametric t-test. (PDF $260 \mathrm{~kb}$ )

Additional file 11: Table S5. Unweighted and weighted UniFrac PERMANOVA p-values of samples from juveniles before and during the HFD challenge (un15mo, un26mo, P4M, HFD). (PDF $12 \mathrm{~kb}$ )

Additional file 12: Figure S7. Alterations in bacterial taxa and metabolic pathways are associated with HFD challenge. A. LEfSe generated cladogram of taxonomic differences of abundance. Areas shaded blue indicate bacterial taxa with a higher abundance in prior synbiotic supplemented animals at 4-months post- supplementation, whereas areas shaded purple indicate bacterial taxa with a higher abundance in prior synbiotic supplemented animals during HFD challenge. B. LEfSe plot of bacterial metabolic pathways with differential abundance. Areas to the right and shaded blue indicate bacterial metabolic pathways enriched in synbiotic-supplemented animals at 4-months post-synbiotic, whereas areas to the left and shaded purple indicate bacterial metabolic pathways enriched in prior synbiotic supplemented animals during HFD challenge. C. LEfSe generated cladogram of taxonomic differences of abundance. Areas shaded blue indicate bacterial taxa with a higher abundance in prior synbiotic supplemented animals at 4-months posttreatment, whereas areas shaded green indicate bacterial taxa with a higher abundance in untreated animals during HFD challenge. D. LEfSe plot of bacterial metabolic pathways with differential abundance. Areas to the right and shaded blue indicate inferred bacterial metabolic pathways enriched in synbiotic supplemented animals at 4-months post-treatment, whereas areas to the left and shaded green indicate bacterial metabolic pathways enriched in untreated animals during HFD challenge. (PDF 3901 kb)
Additional file 13: Figure S8. Relative abundance of LEfSe-identified enriched KEGG pathways in the synbiotic supplemented and untreated groups during HFD challenge. STAMP generated two groups extended error bar plot. Plots on the left indicate relative abundance of enriched KEGG pathways. Plot on the right indicates the difference in the mean proportion. Statistical significance was tested using White's non-parametric t-test. (PDF $461 \mathrm{~kb}$ )

\section{Abbreviations}

HFD: High-fat diet; NAFLD: Non-alcoholic fatty liver disease; NHP: Nonhuman primate; OTU: Operational Taxonomic Unit; SCFA: Short-chain fatty acid

\section{Acknowledgements}

The authors also wish to thank J. Burton for providing materials and reagents, and P. Ganesh and M. Seferovic, for assistance in performing experiments.

\section{Funding}

This research was carried out while R.M.P. was supported through NIH K12 GM084897, A.L.P. was supported through NICHD F32 HD082969, B.D.W.B. was supported through the Cancer Prevention Research Institute of Texas, A.S.H. was supported through the American Chemical Society and Project SEED, and K.M.A. and the work on this project was supported through NIDDK NICHD 1RO1DK089201, R24DK090964, and NIH Director New Innovator Pioneer Award DP21DP2OD001500. The funding bodies had no role in the design of the study and collection, analysis, and interpretation of data and in writing the manuscript.

\section{Availability of data and materials}

The $16 \mathrm{~S}$ sequence data generated from this analysis have been deposited in the Sequence Read Archive (SRA) under accession bioproject ID PRJNA317339.

\section{Authors' contributions}

RMP performed experiments, analyzed the data and authored the manuscript. ALP performed experiments, analyzed the data, and authored the manuscript. J.M. analyzed data, provided computational support and authored the manuscript. KMA designed the studies, provided experimental and analytic oversight, data interpretation and analytic projection, and authored the manuscript. BDWB, ASH, MH, KB, PB, DT, TD, ELS, JEF, PK, and KG contributed to experimental design, maintenance of the animals, sample collection, and provided critical revisions of the manuscript. All authors read and approved the final manuscript.

\section{Ethics approval}

Animals were socially housed within indoor and outdoor enclosures at the Oregon National Primate Research Center (ONPRC). All methods were carried out in accordance with IACUC guidelines and regulations, and all experimental protocols were approved by the IACUC at both ONPRC and Baylor College of Medicine.

\section{Consent for publication}

Not applicable

\section{Competing interests}

The authors declare that they have no competing interests.

\section{Publisher's Note}

Springer Nature remains neutral with regard to jurisdictional claims in published maps and institutional affiliations.

\section{Author details}

${ }^{1}$ Department of Obstetrics and Gynecology, Division of Maternal-Fetal Medicine, Baylor College of Medicine, Houston, TX 77030, USA. ²Department of Molecular and Cell Biology, Baylor College of Medicine, Houston, TX 77030, USA. ${ }^{3}$ Department of Molecular and Human Genetics, Baylor College of Medicine, Houston, TX 77030, USA. ${ }^{4}$ Oregon National Primate Research Center, Oregon Health \& Science University, Beaverton, OR 97239, USA. ${ }^{5}$ Biology Department, University of Portland, Portland, OR 97203, USA. 
${ }^{6}$ Department of Pediatrics, University of Colorado Anschutz Medical Campus, Aurora, CO, USA.

\section{Received: 9 October 2017 Accepted: 19 March 2018 Published online: 05 April 2018}

\section{References}

1. The Human Microbiome Project Consortium. Structure, function and diversity of the healthy human microbiome. Nature. 2012;486:207-14.

2. Ley RE, Turnbaugh PJ, Klein S, Gordon J. Microbial ecology: human gut microbes associated with obesity. Nature. 2006:444:1022-3.

3. Turnbaugh PJ, et al. A core gut microbiome in obese and lean twins. Nature. 2009;457:480-4.

4. David LA, et al. Diet rapidly and reproducibly alters the human gut microbiome. Nature. 2013;505:559-63.

5. Yatsunenko T, et al. Human gut microbiome viewed across age and geography. Nature. 2012;486:222-7.

6. Wu GD, et al. Linking long-term dietary patterns with gut microbial enterotypes. Science. 2011;334:105-8.

7. Walker AW, et al. Dominant and diet-responsive groups of bacteria within the human colonic microbiota. ISME J. 2011;5:220-30.

8. Schnorr SL, et al. Gut microbiome of the Hadza hunter-gatherers. Nat Commun. 2014:5:3654

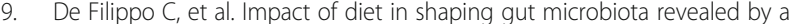
comparative study in children from Europe and rural Africa. PNAS. 2010;107: 14691-6.

10. Hildebrandt MA, et al. High-fat diet determines the composition of the murine gut microbiome independently of obesity. Gastroenterology. 2009; 137:1716-24. e1-2.

11. Ley RE, et al. Obesity alters gut microbial ecology. PNAS. 2005;102:11070-5

12. Turnbaugh PJ, et al. The effect of diet on the human gut microbiome: a metagenomic analysis in humanized gnotobiotic mice. Sci Transl Med. 2009:1:6ra14.

13. Liu S, et al. The effect of diet and host genotype on ceca microbiota of Japanese quail fed a cholesterol enriched diet. Front Microbiol. 2015;6:1092.

14. Lecomte $\mathrm{V}$, et al. Changes in gut microbiota in rats fed a high fat diet correlate with obesity-associated metabolic parameters. PLoS One. 2015;10:e0126931.

15. Serino $\mathbf{M}$, et al. Metabolic adaptation to a high-fat diet is associated with a change in the gut microbiota. Gut. 2012;61:543-53.

16. Sonnenburg ED, et al. Diet-induced extinctions in the gut microbiota compound over generations. Nature. 2016;529:212-5.

17. Parks BW, et al. Genetic control of obesity and gut microbiota composition in response to high-fat, high-sucrose diet in mice. Cell Metab. 2013:17:141-52.

18. Ma J, et al. High-fat maternal diet during pregnancy persistently alters the offspring microbiome in a primate model. Nat Commun. 2014:5:3889.

19. Saha DC, Reimer RA. Long-term intake of a high prebiotic fiber diet but not high protein reduces metabolic risk after a high fat challenge and uniquely alters gut microbiota and hepatic gene expression. Nutr Res. 2014;34:789-96.

20. Qin J, et al. A metagenome-wide association study of gut microbiota in type 2 diabetes. Nature. 2012;490:55-60.

21. Larsen $\mathrm{N}$, et al. Gut microbiota in human adults with type 2 diabetes differs from non-diabetic adults. PLoS One. 2010;5:e9085.

22. Ridaura VK, et al. Gut microbiota from twins discordant for obesity modulate metabolism in mice. Science. 2013:341:1241214.

23. Turnbaugh PJ, et al. An obesity-associated gut microbiome with increased capacity for energy harvest. Nature. 2006:444:1027-31.

24. Pound LD, Comstock SM, Grove KL. Consumption of a western-style diet during pregnancy impairs offspring islet vascularization in a Japanese macaque model. Am J Phys Metab. 2014;307:E115-23.

25. Thorn SR, et al. Early life exposure to maternal insulin resistance has persistent effects on hepatic NAFLD in juvenile nonhuman primates Diabetes. 2014;63:2702-13.

26. Fuller R. Probiotics in man and animals. J Appl Bacteriol. 1989;66:365-78.

27. Gibson GR, Roberfroid MB. Dietary modulation of the human colonic microbiota: introducing the concept of prebiotics. J Nutr. 1995:125:1401-12.

28. Tanaka $R$, et al. Effects of administration of TOS and Bifidobacterium breve 4006 on the human fecal flora. Bifidobacteria Microflora. 1983:2:17-24.

29. Bouhnik $Y$, et al. Effects of Bifidobacterium sp fermented milk ingested with or without inulin on colonic bifidobacteria and enzymatic activities in healthy humans. Eur J Clin Nutr. 1996;50:269-73.
30. Bouhnik Y, et al. Four-week short chain fructo-oligosaccharides ingestion leads to increasing fecal bifidobacteria and cholesterol excretion in healthy elderly volunteers. Nutr J. 2007;6:1-7.

31. Roberfroid MB, Van Loo JA, Gibson GR. The bifidogenic nature of chicory inulin and its hydrolysis products. J Nutr. 1998;128:11-9.

32. Boehm G, et al. Supplementation of a bovine milk formula with an oligosaccharide mixture increases counts of faecal bifidobacteria in preterm infants. Arch Dis Child Fetal Neonatal Ed. 2002;86:F178-81.

33. Bomhof MR, Saha DC, Reid DT, Paul HA, Reimer RA. Combined effects of oligofructose and Bifidobacterium animalis on gut microbiota and glycemia in obese rats. Obesity. 2014;22:763-71.

34. Bartosch S, Woodmansey EJ, Paterson JCM, McMurdo MET, Macfarlane GT. Microbiological effects of consuming a synbiotic containing Bifidobacterium bifidum, Bifidobacterium lactis, and oligofructose in elderly persons, determined by real-time polymerase chain reaction and counting of viable bacteria. Clin Infect Dis. 2005;40:28-37.

35. Fujimori $\mathrm{S}$, et al. A randomized controlled trial on the efficacy of synbiotic versus probiotic or prebiotic treatment to improve the quality of life in patients with ulcerative colitis. Nutrition. 2009;25:520-5.

36. Bakker-Zierikzee AM, et al. Effects of infant formula containing a mixture of galacto- and fructo-oligosaccharides or viable Bifidobacterium animalis on the intestinal microflora during the first 4 months of life. Br J Nutr. 2005;94:783-90.

37. Cox J, Williams S, Grove K, Lane RH, Aagaard-Tillery KM. A maternal high-fat diet is accompanied by alterations in the fetal primate metabolome. Am J Obstet Gynecol. 2009;201(281):e1-9.

38. McCurdy $C E$, et al. Maternal high-fat diet triggers lipotoxicity in the fetal livers of nonhuman primates. J Clin Invest. 2009;119:323-35.

39. Suter MA, et al. Maternal high-fat diet modulates the fetal thyroid axis and thyroid gene expression in a nonhuman primate model. Mol Endocrinol. 2012;26:2071-80.

40. Fischer $\mathrm{MH}$, et al. The gel-forming polysaccharide of psyllium husk (Plantago ovata Forsk). Carbohydr Res. 2004:339:2009-17.

41. Fujimori $\mathrm{S}$, et al. High dose probiotic and prebiotic cotherapy for remission induction of active Crohn's disease. J Gastroenterol Hepatol. 2007:22:1199-204.

42. Rishniw M, Wynn SG. Azodyl, a synbiotic, fails to alter azotemia in cats with chronic kidney disease when sprinkled onto food. J Feline Med Surg. 2011; 13:405-9.

43. Belknap D, Davidson LJ, Smith CR. The effects of psyllium hydrophilic mucilloid on diarrhea in enterally fed patients. Heart Lung. 1997;26:229-37.

44. Smalley JR, Klish WJ, Campbell MA, Brown MR. Use of psyllium in the management of chronic nonspecific diarrhea of childhood. J Pediatr Gastroenterol Nutr. 1982;1:361-3.

45. Singh B. Psyllium as therapeutic and drug delivery agent. Int J Pharm. 2007; 334:1-14.

46. Songer JG. Clostridial enteric diseases of domestic animals. Clin Microbiol Rev. 1996;9:216-34.

47. Aagaard-Tillery KM, et al. Developmental origins of disease and determinants of chromatin structure: maternal diet modifies the primate fetal epigenome. J Mol Endocrinol. 2008:41:91-102.

48. Suter MA, et al. A maternal high-fat diet modulates fetal SIRT1 histone and protein deacetylase activity in nonhuman primates. FASEB. 2012;26:5106-14.

49. Suter M, et al. Epigenomics: maternal high-fat diet exposure in utero disrupts peripheral circadian gene expression in nonhuman primates. FASEB. 2011;25:714-26

50. Suter MA, Takahashi D, Grove KL, Aagaard KM. Postweaning exposure to a high-fat diet is associated with alterations to the hepatic histone code in Japanese macaques. Pediatr Res. 2013;74:252-8.

51. Aagaard KM, et al. A metagenomic approach to characterization of the vaginal microbiome signature in pregnancy. PLoS One. 2012;7:e36466-15.

52. Caporaso JG, et al. QIIME allows analysis of high-throughput community sequencing data. Nat Meth. 2010;7:335-6.

53. DeSantis TZ, et al. Greengenes, a chimera-checked 16S rRNA gene database and workbench compatible with ARB. Appl Environ Microbiol. 2006;72: 5069-72.

54. Lozupone C, Hamady M, Knight R. UniFrac-an online tool for comparing microbial community diversity in a phylogenetic context. BMC Bioinformatics. 2006;7:371.

55. Langille MG, et al. Predictive functional profiling of microbial communities using 16S rRNA marker gene sequences. Nat Biotechnol. 2013;31:814-21.

56. Parks DH, Tyson GW, Hugenholtz P, Beiko RG. STAMP: statistical analysis of taxonomic and functional profiles. Bioinformatics. 2014;30:3123-4. 
57. Segata N, et al. Metagenomic biomarker discovery and explanation. Genome Biol. 2011;12:R60.

58. Cheng S, et al. A PCR assay for identification of enterococcus faecium. J Clin Microbiol. 1997:35:1248-50

59. Pérez-Díaz IM. Putative and unique gene sequence utilization for the design of species specific probes as modeled by lactobacillus plantarum. Curr Microbiol. 2013;66:266-70.

60. Ye J, et al. Primer-BLAST: a tool to design target-specific primers for polymerase chain reaction. BMC Bioinformatics. 2012;13:134.

61. Singh KM, et al. Study of rumen metagenome community using qPCR under different diets. Meta Gene. 2014;2:191-9.

62. Haro C, et al. The gut microbial community in metabolic syndrome patients is modified by diet. J.Nutr. Biochemist. 2016;27:27-31.

63. Fujimoto $T$, et al. Decreased abundance of Faecalibacterium prausnitzii in the gut microbiota of Crohn's disease. J Gastroenterol Hepatol. 2013; 28:613-9.

64. Sokol $\mathrm{H}$, et al. Faecalibacterium prausnitzii is an anti-inflammatory commensal bacterium identified by gut microbiota analysis of Crohn disease patients. PNAS. 2008:105:16731-6.

65. Singh KM, et al. Molecular genetic diversity and quantitation of methanogen in ruminal fluid of buffalo (Bubalus bubalis) fed ration (wheat straw and concentrate mixture diet). Genet Res Int. 2013;2013:980191.

66. Schaafsma G, Meuling W, Van Dokkum W, Bouley C. Effects of a milk product, fermented by lactobacillus acidophilus and with fructooligosaccharides added, on blood lipids in male volunteers. Euro J Clinical Nutr. 1998:52:436-40

67. Esposito $E$, et al. Probiotics reduce the inflammatory response induced by a high-fat diet in the liver of young rats. J Nutr. 2009;139:905-11.

68. An D, et al. Sphingolipids from a symbiotic microbe regulate homeostasis of host intestinal natural killer T cells. Cell. 2014;156:123-33.

69. Suen $\mathrm{G}$, et al. The complete genome sequence of Fibrobacter succinogenes S85 reveals a cellulolytic and metabolic specialist. PLoS One. 2011;6:e18814.

70. Esteve $M$, et al. Intestinal spirochetosis and chronic watery diarrhea: clinical and histological response to treatment and long-term follow up. J Gastroenterol Hepatol. 2006;21:1326-33.

71. Caro-Quintero A, Ritalahti KM, Cusick KD, Loffler FE, Konstantinidis KT. The chimeric genome of Sphaerochaeta. MBio. 2012;3:e00025-12.

72. Dewhirst FE, et al. 'Flexispira rappini' strains represent at least 10 Helicobacter taxa. Int J Syst Evol Microbiol. 2000;50:1781-7.

73. Haines DC, et al. Inflammatory large bowel disease in immunodeficient rats naturally and experimentally infected with Helicobacter bilis. Vet Pathol. 1998:35:202-8

74. Downes J, et al. Bulleidia extructa gen. Nov., sp. nov., isolated from the oral cavity. Int J System Evol Microbiol. 2000;50:979-83.

75. Jackson HT, et al. Culture-independent evaluation of the appendix and rectum microbiomes in children with and without appendicitis. PLOS One. 2014:9:e95414

76. Louis P, Flint HJ. Diversity, metabolism and microbial ecology of butyrateproducing bacteria from the human large intestine. FEMS Microbiol Lett. 2009;294:1-8.

77. Naka S, et al. A specific Streptococcus mutans strain aggravates nonalcoholic fatty liver disease. Oral Dis. 2014;20:700-6.

78. Grant WF, et al. Maternal high fat diet is associated with decreased plasma n-3 fatty acids and fetal hepatic apoptosis in nonhuman primates. PLoS One. 2011:6:e17261.

79. Prince $A L$, et al. The placental membrane microbiome is altered among subjects with spontaneous preterm birth with and without chorioamnionitis. Am J Obstet Gynecol. 2016;214:627.e1-627-e16.

80. Eren AM, Borisy GG, Huse SM, Mark Welch JL. Oligotyping analysis of the human oral microbiome. PNAS. 2014;111:E2875-84.

81. Romero $\mathrm{R}$, et al. The composition and stability of the vaginal microbiota of normal pregnant women is different from that of non-pregnant women. Microbiome. 2014;2:4.

82. Dean JT, et al. Resistance to diet-induced obesity in mice with synthetic Glyoxylate shunt. Cell Metab. 2009;9:525-36

83. den Besten $\mathrm{G}$, et al. The role of short-chain fatty acids in the interplay between diet, gut microbiota, and host energy metabolism. J Lipid Res. 2013;54:2325-40

84. Kaur A, Rose DJ, Rumpagaporn P, Patterson JA, Hamaker BR. In vitro batch fecal fermentation comparison of gas and short-chain fatty acid production using 'slowly fermentable' dietary fibers. J Food Sci. 2011;76:H137-42.
85. Chan M-Y, Heng C-K. Sequential effects of a high-fiber diet with psyllium husks on the expression levels of hepatic genes and plasma lipids. Nutrition. 2008;24:57-66.

86. Tang $Y$, Saris PEJ. Strain-specific detection of orally administered canine jejunum-dominated lactobacillus acidophilus LAB20 in dog faeces by realtime PCR targeted to the novel surface layer protein. Lett Appl Microbiol. 2013;57:330-5.

87. Uyeno $Y$, Sekiguchi $Y$, Kamagata $Y$. Impact of consumption of probiotic lactobacilli-containing yogurt on microbial composition in human feces. Int J Food Microbiol. 2008;122:16-22.

88. Abdulkadir $\mathrm{B}$, et al. Routine use of probiotics in preterm infants: longitudinal impact on the microbiome and metabolome. Neonatology. 2016;109:239-47.

89. Brooks $B$, et al. Microbes in the neonatal intensive care unit resemble those found in the gut of premature infants. Microbiome. 2014;2:1.

90. Ríos-Covián $\mathrm{D}$, et al. Intestinal short chain fatty acids and their link with diet and human health. Front Microbiol. 2016:7:2030

\section{Submit your next manuscript to BioMed Central and we will help you at every step:}

- We accept pre-submission inquiries

- Our selector tool helps you to find the most relevant journal

- We provide round the clock customer support

- Convenient online submission

- Thorough peer review

- Inclusion in PubMed and all major indexing services

- Maximum visibility for your research

Submit your manuscript at www.biomedcentral.com/submit
Biomed Central 